\title{
A rare tumor of the lung, hepatoid adenocarcinoma
}

\begin{abstract}
The hepatoid adenocarcinoma is an uncommon tumor and even more uncommon whenit is located in the pulmonary system. This neoplasm is highly related to the synthesis of alphafetoprotein. We present a case of a 60 year old male patient who presented uncontrolled chronic pain on his left shoulderand left chest. After few studies, in an antero-posterior $\mathrm{X}$ ray of the shoulder, a pulmonary mass was found. The mass was then diagnosed with the corresponding studiesas a pulmonary hepatoid adenocarcinoma. In this report we mention the characteristics of this pathology in different organs and systems, mainly in the respiratory system.
\end{abstract}

Keywords: hepatoidadenocarcinoma, tumor, hepatocellular, cancer, neoplasm, alphafetoprotein, chemotherapy
Volume 10 Issue $3-2020$

\author{
Salomon Jasqui Remba, Isaac Baley Amiga, \\ Alan Jasqui Bucay \\ Centro Médico ABC,Anahuac University, Mexico
}

Correspondence: Dr Salomon Jasqui-Remba, Affiliation, Av Carlos Fernández Graef I54, Santa Fe, Office \#073, Contadero, Cuajimalpa de Morelos, 05330 Mexico, Tel 5I229090, Emaildrsjasqui@gmail.com

Received: April 18, 2020 | Published: May 13, 2020

\section{Introduction}

Hepatoid adenocarcinoma is a rare neoplasm, ${ }^{1}$ named for its histopathological and immunohistochemical similarity with the hepatocellular carcinoma. The extrahepatic localization has a poor prognosis and tends to occur more frequently in the stomach. An important characteristic is the ability to produce alpha-fetoprotein, like many other pulmonary neoplasms. Its ability to respond to treatment and the possibility of recurrence is another relevant characteristic., ${ }^{2,3}$

\section{Case presentation}

This is a case of a 60 year old man which presented with uncontrollable left shoulder, arm, hand and chest pain. The symptoms were manifested at rest or with hardly any movement. The patient refers taking over the counter analgesic treatment without improvement.

The patient consulted with a cardiology team where an electrocardiogram and an exercise test were performed, being reported normal. He was then referred to the orthopedics service due to shoulder pain persistence. A shoulder x-ray showed a tumor in the left thorax measuring $78.99 \mathrm{~mm}$ in diameter (Figure 1). In follow up studies, a left humeral lesion of neoplastic appearance was revealed. In the magnetic resonance an intramedullary lesion is reported in the sternum, incidentally, a solid mass is observed in the left lung, it also shows tendinitis of the rotator cuff and subacromial impingement (Figures 2-4). Therefore, it is decided to perform a Computed Tomography scan which reported atelectasis mainly in the left upper lobe and a tumor measuring $65 \mathrm{~mm}$ (Figure 5). The Positron Emission Tomography - Computed Tomography scan result was an increase of the metabolism by neoplastic activity, lymphadenopathy and bone lesions, hepatosplenomegaly and non- specific alterations in the colon (Figure 6).

Pathology studies showed a malignant neoplasia focal expression of CK7 in the lung, diffuse expression of CK AE 1/3, in addition to Hepart-1, arginase in neoplastic cells, cytoplasmic TTF-1 (nonnuclear), positive CDX2. The final histopathological result was a left lung tumor with hepatoid differentiation.
The values reported of alpha-fetoprotein where $716.5(\mathrm{ng} / \mathrm{ml}$ (normal ranges from 0 to $15 \mathrm{ng} / \mathrm{ml}$ ) and carcinoembryonic antigen values where $53.7 \mathrm{ng} / \mathrm{ml}$ (normal ranges from 0 to $3 \mathrm{ng} / \mathrm{ml}$ ).

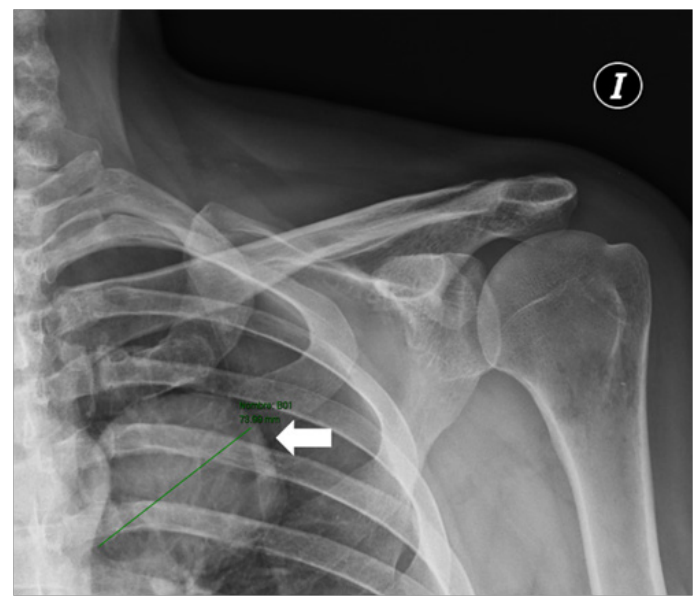

Figure I Shoulder x-ray showed a tumor in the left thorax measuring $78.99 \mathrm{~mm}$ in diameter.

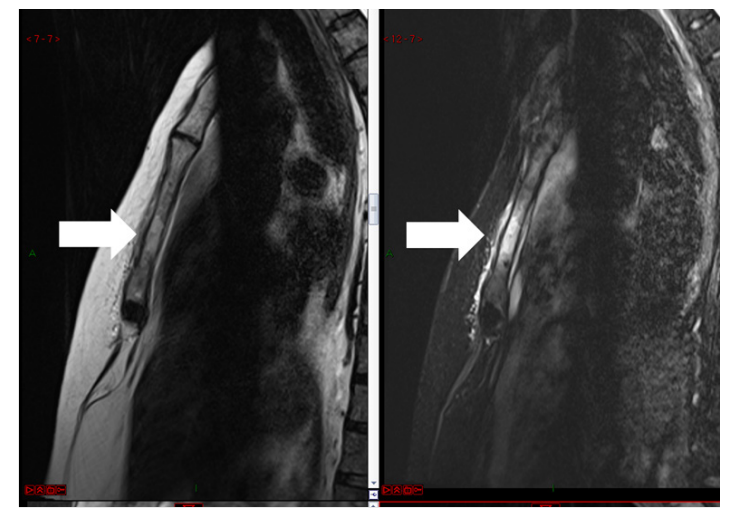

Figure 2 Magnetic resonance with intramedullary lesion in the sternum. 


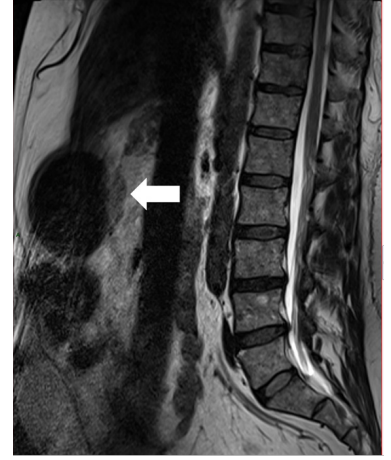

Figure 3 Magnetic resonance with a solid mass observed in the left lung.

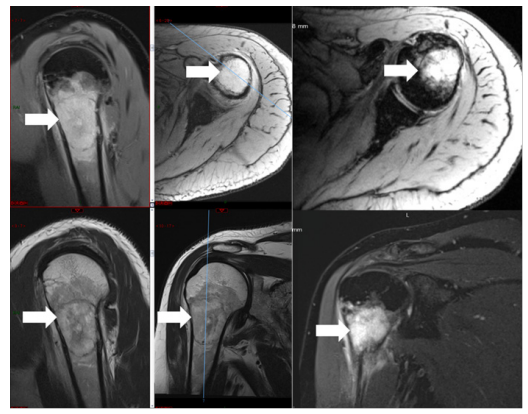

Figure 4 Magnetic resonance shows a left humeral lesion of neoplastic appearance.

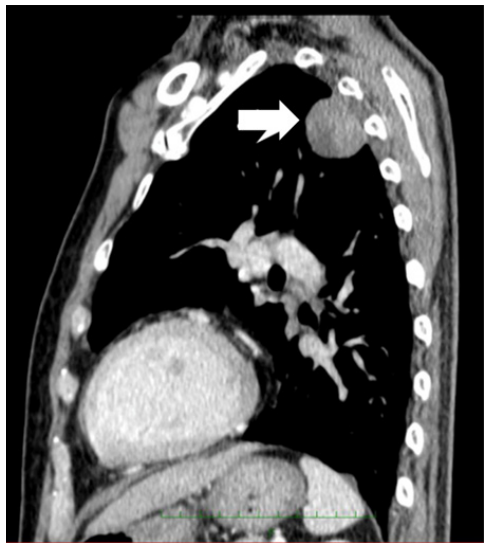

Figure $\mathbf{5}$ Chest CT.A mass shadow is present in the left upper lung field.

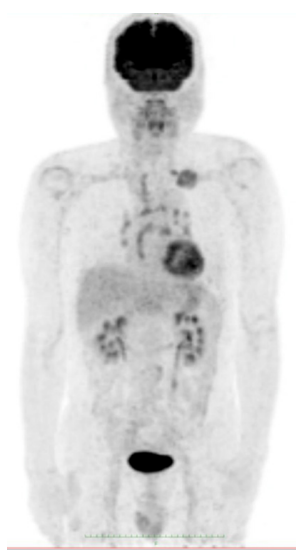

Figure 6 Fluorodeoxyglucose-positron emission tomography shows abnormal uptake in the left lung tumor, bilateral hilar lymph nodes, and left supraclavicular lymph nodes.

\section{Investigations}

Hepatoid carcinoma was described in 1985 by Ishikura et al., ${ }^{1}$ as a rare malignant tumor that mimics the characteristics of metastatic hepatocellular carcinoma to the lung. Initially it was described as a gastric tumor due to the percentage of appearance in the gastric system. It most commonly occurs in the stomach $(63 \%)$, ovaries $(10 \%)$, lungs $(5 \%)$, gallbladder $(4 \%)$, pancreas $(4 \%)$ and uterus (4\%). ${ }^{4}$ It's predominant in older men and closely related to a history of heavy smoking and has a very poor prognosis, with an average survival rate of 11 months after the diagnosis is made. Almost all hepatoid carcinoma expresses alpha-fetoprotein, making it such a distinguishable characteristic that some authors make it indispensable for the diagnosis, however, not all tumors produce it. ${ }^{3,5}$ Histologically has hepatocellular characteristics with a pseudo-biliary pattern, in addition multiple immunophenotypes have been described, among them HepPar1, CK7, EpCAM antibody HEA125 or MOC31; these differentiate them from hepatocytes and support the diagnosis (mainly in cases where there is absence of alpha-fetoprotein production). ${ }^{5}$

It is commonly found as a solid tumor located in a pulmonary apex with lymph node involvement, distant metastasis and uptake of 18FDG.

\section{Differential diagnosis}

The differential diagnosis of these pulmonary tumors secreting alpha-fetoprotein must be made with lung metastases from other hepatoid adenocarcinomas, especially hepatocellular carcinoma, ovarian and gastric cancer and with mediastinal germ cell tumors, usually more undifferentiated, without features of hepatocellular differentiation and with secretion of human chorionic gonadotropin. ${ }^{3}$

\section{Treatment}

Chemotherapy was based on two cycles of a scheme of Carboplatin AUC 5+ Pemetrexed, which was then changed to pembrolizumab and radiotherapy, he received 5 sessions in chest and 5 sessions in humerus. In addition, pain control drugs were administered. Despite the therapeutic efforts, the prognosis for this disease is poor.

\section{Results}

The patient was hospitalized for pain control. In the hospital he suffered a convulsive crisis, respiratory distress and cardiac arrest. The final diagnosis was non-small cell lung cancer with hepatoid differentiation.

\section{Discussion}

There are few reports and studies of hepatoid adenocarcinoma due to its low incidence. This type of tumor is uncommon in the lung, corresponding to less than $5 \%$ of the total hepatoid adenocarcinoma. The first tumor of this kind was found as a gastric tumor, being the most common type of hepatoid adenocarcinoma. Ishikura et al., ${ }^{1}$ also report the important relationship between this tumor and the production of alpha-fetoprotein, so much that some authors take it into account as a criterion for its diagnosis, however, Grossman et al, concluded that the existence of alpha-fetoprotein is not necessary for the diagnosis, because of the existence of other criteria. Most published studies agree with the poor prognosis of this disease despite subjecting patients to the indicated treatment.

The case patient started with pain symptoms due to bone lesions caused by tumor growth. The pain persistence was the key 
element to identify the disease. However, despite the diagnostic and right treatment, the prognosis for this tumor is poor as previously mentioned, and the patient died months after diagnosis.

\section{Conclusion}

Hepatoid adenocarcinoma is a rare neoplasm, manly in the GI system but it could also present itself in the lung and other systems, and has poor prognosis, despite the treatment.. This tumoritis highly related with alpha-feto protein production.

\section{Acknowledgments}

None.

\section{Funding}

None.

\section{Conflicts of interest}

The authors have no conflicts of interest to declare.

\section{References}

1. Ishikura H, Kanda M, Ito M, et.al. Hepatoid adenocarcinoma: Adistinctive histological subtype of alpha-fetoprotein-producing lung carcinoma. Virchows Archiv A Pathol Anat. 1990;417(1):73-80.

2. Motooka Y, Yoshimoto K, Semba T, et al. Pulmonary hepatoid adenocarcinoma: report of a case. Motooka et al. Surgical Case Reports. 2016;2:1.

3. Vidal L, Casado E, Pallarés MC, et al. Tumor pulmonar productor de alfafetoproteína: a propósito de dos casos. Rev Oncol. 2003;5(2):113-4.

4. Metzgeroth G, Ströbel P, Baumbusch T, et al., Hepatoid adenocarcinoma - review of the literature illustrated by a rare case originating in the peritoneal cavity. Onkologie. 2010;33(5):263-269.

5. Grossman K, Beasley MB, Braman SS. Braman. Hepatoid adenocarcinoma of the lung: Review of a rare form of lung cancer. Respir Med. 2016;175-179. 